\title{
Visual Outcomes after Bilateral Implantation of an Extended Depth of Focus Intraocular Lens: a Multicenter Study
}

\author{
Moonjung Choi ${ }^{1}$, Chan Young Im$^{2}$, Jin Ki Lee ${ }^{3}$, Hyung il Kim ${ }^{4}$, Hyo Soon Park ${ }^{5}$ and Tae im Kim ${ }^{6 *}$ \\ ${ }^{1}$ Department of Ophthalmology, Konyang University College of Medicine, Konyang University Myunggok Medical Research Center, Korea \\ ${ }^{2}$ Ian Eye Center, Korea \\ ${ }^{3}$ Daegu Fatima Hospital, Korea \\ ${ }^{4}$ Gyeongju St. Mary's Eye Clinic, Korea \\ ${ }^{5}$ Nunevit Eye Center, Korea \\ ${ }^{6}$ Department of Ophthalmology, Severance Hospital, Yonsei University College of Medicine, Korea \\ *Corresponding author: Tae im Kim, Department of Ophthalmology, Severance Hospital, Yonsei University College of Medicine, Korea
}

\begin{abstract} (IOL).

Purpose: To evaluate the visual performance after bilateral implantation of an extended depth of focus (EDOF) intraocular lens

Methods: In this multicenter, prospective, observational study, the postoperative outcomes of 96 patients who underwent bilateral cataract surgery with EDOF IOL (TECNIS Symfony) were assessed.

Results: Mean decimal visual acuity results showed a binocular corrected distance visual acuity (CDVA) of $1.10 \pm 0.18$, uncorrected distance visual acuity (UDVA) of $1.04 \pm 0.17$, uncorrected intermediate visual acuity (UIVA) of $0.96 \pm 0.16$, and uncorrected near visual acuity (UNVA) of $0.68 \pm 0.18$. Overall, $76 \%$ of the patients achieved spectacle independence across all distances, and more than $85 \%$ reported no or mild dysphotoptic phenomena. On a scale from 0 to 10 , median patient satisfaction score was 9 for far, 9.5 for intermediate, and 8 for near vision.

Conclusions: The Symfony EDOF IOL provided excellent distance and intermediate visual outcome and functional near visual acuity. The visual results were associated with high levels of spectacle independence and patient satisfaction.

Keywords: Extended Depth of Focus; Multifocal Intraocular Lens; Presbyopia; Cataract Extraction; Intraocular Lens Implantation Abbreviations

EDOF: Extended depth of focus; IOL: Intraocular lens; CDVA: Corrected Distance Visual Acuity; UDVA: Uncorrected Distance Visual Acuity; UIVA: Uncorrected Intermediate Visual Acuity; UNVA: Uncorrected Near Visual Acuity; MRSE: Manifest Refraction Spherical Equivalent
\end{abstract}

\section{Introduction}

With increased patients' demand for a spectacle-free lifestyle and technological advancements, cataract surgery with multifocal intraocular lens (IOL) implantation has become an effective solution for correcting presbyopia in patients who want to maintain their full range of vision. Conventional bifocal IOLs used either refractive or diffractive optics to split the light and create 2 principal focal points, thus providing functional vision at distance and near [1]. This simultaneous imaging principle produces a 
sharp image that is overlaid by a secondary blurred out of focus image, so these IOLs inherently produce a deterioration in contrast sensitivity and unwanted photic phenomena such as glare and halos [2]. Additionally, studies reported insufficient intermediate vision with bifocal IOLs $[3,4]$. There is an increasing desire for spectacle independence at intermediate distance, especially due to the increased use of computers and smartphones. Trifocal IOLs were introduced to the market in 2010 and they provide an additional, third focal point to improve intermediate vision without compromising distance and near vision [5]. However, they are still vulnerable to decreased contrast sensitivity and photic phenomena due to the IOL's mechanism of splitting the light into discrete focal points [5]. An extended depth of focus (EDOF) technology was recently introduced with the aim to improve intermediate vision while maintaining image contrast. The diffractive echelette design, embedded on its posterior optical surface, extends the range of vision and the achromatic technology reduces corneal chromatic aberrations to enhance retinal image quality and improve contrast sensitivity [6,7]. The elongated focus allows imaging in a continuous range of vision without overlapping near and far images, and therefore theoretically would provide a more consistent distance and intermediate vision with less dysphotopsia. The aim of this multicenter study was to investigate the clinical outcome of patients bilaterally implanted with either Tecnis Symfony or Tecnis Symfony toric extended range of vision IOLs (Johnson \& Johnson Vision, Santa Ana, CA, USA) in terms of visual performance at different distances, spectacle independence, photic phenomena, and patient satisfaction.

\section{Materials and Methods}

\section{Study design and patients}

This 6-month, multi-center, prospective, non-comparative, observational study was conducted at 5 participating clinical sites in South Korea. It included patients who had uncomplicated cataract surgery with binocular implantation of either Symfony (ZXR00) or Symfony toric (ZXT) IOLs from June 2016 to June 2018. The non-toric Symfony IOL was implanted in eyes with a corneal astigmatism of less than 1.0 diopters (D). In cases of a regular corneal astigmatism of greater than $1.0 \mathrm{D}$, the Symfony toric ZXT was recommended. Target refraction was emmetropia for all patients. Follow-up examinations were performed 1 day, 1 week, 4-8 weeks, and 4-6 months postoperatively. Exclusion crite $\neg$ ria were the presence of amblyopia, keratoconus, previous corneal or refractive surgery, chronic or recurrent uveitis, acute ocular diseases, previous ocular surgeries, glaucoma, and any ocular diseases which could possibly affect the postoperative visual acuity. This study was performed in accordance with the tenets of the Declaration of Helsinki and approved by the Severance Hospital Institutional Review Board, Seoul, South Korea. All patients provided written informed consent before study enrollment. The study was registered at the US National Institutes of Health (ClinicalTrials.gov) \#NCT03997890.

\section{The IOL}

The Tecnis Symfony IOL is a one-piece, ultraviolet-blocking, hydrophobic acrylic foldable lens with an overall length of 13 $\mathrm{mm}$, an optic diameter of $6 \mathrm{~mm}$, and a refractive index of 1.47. It incorporates a frosted edge design with a $360^{\circ}$ posterior square edge. It has a biconvex, wavefront-designed anterior aspheric (-0.27 $\mu \mathrm{m}$; model ZXR00) or toric-aspheric (model ZXT) surface to compensate for corneal spherical aberrations and a posterior achromatic diffractive surface to enhance contrast sensitivity. The echelette design allows a pattern of pupil-dependent light diffraction which elongates the focal zone, resulting in an extended range of vision. It is available in spherical equivalent powers of 5.0 to $34.0 \mathrm{D}$ in $0.5 \mathrm{D}$ increments, and in five cylindrical powers: 1.00 , $1.50,2.25,3.00$, and $3.75 \mathrm{D}$ (at the IOL plane).

\section{Postoperative assessments}

Binocular corrected (CDVA) and uncorrected (UDVA) distance visual acuity was assessed at $4 \mathrm{~m}$ using a Snellen chart. Binocular uncorrected intermediate visual acuity (UIVA) at $70 \mathrm{~cm}$ and binocular uncorrected near visual acuity (UNVA) at $40 \mathrm{~cm}$ were assessed using the Rosenbaum near vision card. A subjective questionnaire on spectacle use, photic phenomena, and satisfaction was administered to all patients. Patients were asked how often (never, occasionally, $50 \%$ of the time, frequently) they wear spectacles for near, intermediate, and distance activities. Nondirected and directed questions were used with regard to visual symptoms. Photic phenomena (halos, glare and starbursts) were graded as none, trace, mild, moderate, or severe. Patients were asked to rate their level of satisfaction with distance, intermediate, and near vision on a scale from 0 (completely dissatisfied) to 10 (completely satisfied). Additionally, they were asked whether they would choose the same IOL again and if they would recommend the IOL to their friends and family. Surgeons were also asked to rate their level of satisfaction on a scale from 0 to 10 in terms of the ease of manipulation and implantation of the IOL and the visual performance of the IOL.

\section{Statistical Analysis}

Summary descriptive statistics were produced for all key variables of the study. Continuous variables were expressed as mean \pm standard deviation and the percentage frequency was calculated for all categorical data.

\section{Results}

\section{Patient demographics and refractive status}

A total of 100 patients who underwent bilateral IOL implantation with either Symfony or Symfony toric IOL were 
enrolled in this study. Ninety-six patients (192 eyes) completed the 4-6 months assessment and were included in the final analysis. The mean age was $61.19 \pm 10.25$ years, and $64.6 \%$ (62 patients) were females. The preoperative mean manifest refraction spherical equivalent (MRSE) was $-0.57 \pm 3.01 \mathrm{D}$. At the last postoperative visit after $4-6$ months the MRSE was $-0.41 \pm 0.44 \mathrm{D}$. The postoperative spherical equivalent was within $0.50 \mathrm{D}$ in $72.4 \%$ of eyes, and $90.6 \%$ of eyes had less than $1.00 \mathrm{D}$ of residual cylinder. Eleven patients $(11.5 \%)$ received the toric Tecnis Symfony IOL in both eyes, and 1 patient $(1.0 \%)$ had a mixed implantation of Symfony and Symfony toric IOLs. The mean age of patients who received the toric version in at least one eye was $54.75 \pm 10.99$ years, and $58.3 \%$ ( 7 patients) were female. The preoperative MRSE was $-1.99 \pm 3.57 \mathrm{D}$, and the postoperative MRSE at 4-6 months was $-0.46 \pm 0.41 \mathrm{D}$.

\section{Visual outcome}

In all patients, mean binocular CDVA was $1.10 \pm 0.18(-0.04 \pm$ $0.07 \operatorname{logMAR})$ and mean binocular UDVA was $1.04 \pm 0.17(-0.02$ $\pm 0.07 \log$ MAR). Mean binocular UIVA and UNVA was $0.96 \pm 0.16$ $(0.04 \pm 0.07 \log \mathrm{MAR})$ and $0.68 \pm 0.18(0.17 \pm 0.14 \log \mathrm{MAR})$, respectively. In patients with toric Symfony IOLs in at least one eye, mean binocular CDVA was $1.09 \pm 0.18(-0.04 \pm 0.07 \log$ MAR); mean binocular UDVA was $1.00 \pm 0.20(0.00 \pm 0.09 \log$ MAR $)$; mean binocular UIVA was $0.92 \pm 0.12(0.04 \pm 0.06 \operatorname{logMAR})$; and mean binocular UNVA was $0.74 \pm 0.18(0.13 \pm 0.12 \operatorname{logMAR})$. The cumulative percentages of patients reaching various visual acuity levels across different distances are shown in Figure 1.

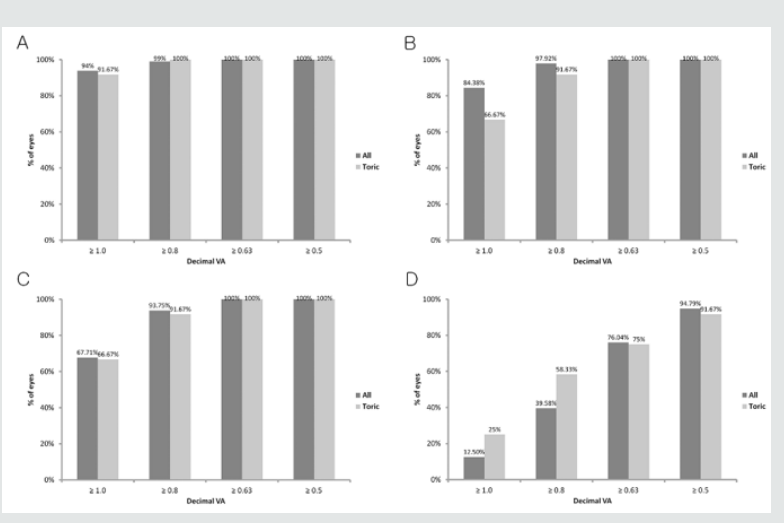

Figure 1: Cumulative binocular visual acuity. (A) Corrected distance visual acuity. (B) Uncorrected distance visual acuity. (C) Uncorrected intermediate visual acuity. (D) Uncorrected near visual acuity.

\section{Spectacle independence}

Overall, $76 \%$ of all patients, and $75 \%$ of toric patients never or only occasionally used spectacles across all distances. Regarding distance vision, $96 \%$ of all patients and $92 \%$ of toric patients stated that they never or only occasionally need glasses (Figure 2). For intermediate tasks, $3.1 \%$ of all patients required spectacles most of the time whereas all of the toric patients were completely spectacle independent. In total, $13.5 \%$ of all patients and $8.3 \%$ of the toric patients frequently needed reading glasses (Figure 2).
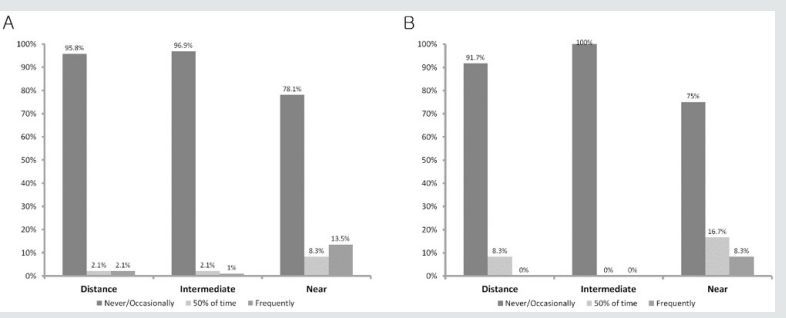

Figure 2: Spectacle independence of all patients (A) and patients implanted with toric IOLs (B).

\section{Photic phenomena}

On nondirected questioning, $23.0 \%$ of patients said that they had some visual symptoms. Overall, $92.0 \%, 89.4 \%$, and $86.7 \%$ of all patients, and $91.7 \%, 91.7 \%$, and $83.3 \%$ of the toric patients reported no or minimal halo, glare, and starburst, respectively (Figure 3).

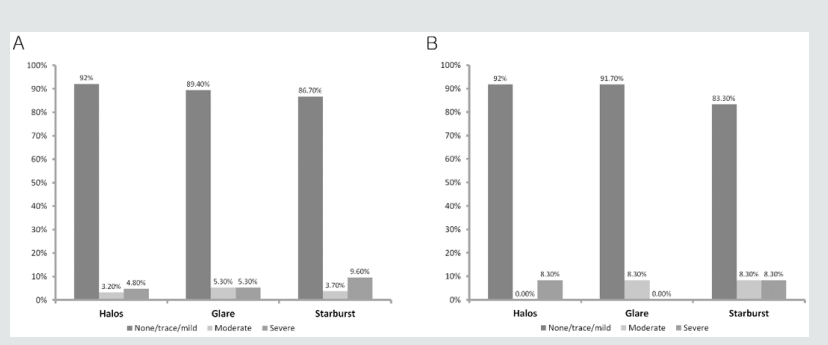

Figure 3: Incidence of photic phenomena of all patients (A) and patients implanted with toric IOLs (B).

\section{Patient and surgeon satisfaction}

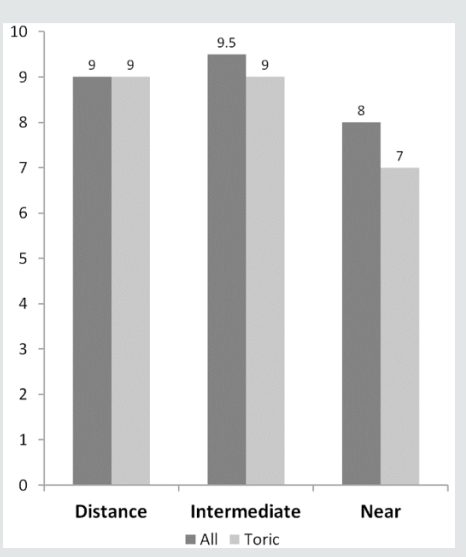

Figure 4: Patient satisfaction score regarding distance, intermediate, and near vision.

The median score for patient satisfaction was 9.0 (range, 5-10) for distance, 9.5 (range, 6-10) for intermediate, and 8.0 (range, 
1-10) for near vision (Figure 4). The median score for toric patients was 9.0 for distance (range, 7-10) and intermediate (range, 6-10), and 7.0 for near vision (range, 4-10) (Figure 4). In total, $81.25 \%$ of patients were willing to recommend the IOL to friends and family, and $80.21 \%$ said that they would choose the same IOL again. The overall surgeon satisfaction was high with a median score of 9.0 (range 6-10), and also all other criteria, including implantation and handling of the IOL, achievement of target refraction and visual performance, reached a median score of 9.0 (range, 6-10).

\section{Complications and adverse events}

One patient had macular edema in the right eye and was treated with posterior subtenon triamcinolone injection after which the eye fully recovered. A refractive touch-up treatment was done in 5 eyes of 5 patients. There was no incidence of IOL decentration or tilt.

\section{Discussion}

The introduction of various multifocal IOLs has offered surgical options for correcting presbyopia in cataract patients who want spectacle independence in daily routine activities. The selection of IOL depends on the patient's characteristics, preferences, lifestyle, job, and vision expectations. Intermediate vision is becoming more important for working life and daily activities due to the increased use of computers, tablets and smartphones. Bifocal IOLs with a low or moderate near addition may help to improve intermediate vision at the expense of near vision, whereas trifocal IOLs offer an additional focus for intermediate vision [8]. However, the principle of multifocality, in which the image is only sharp within a limited zone around the foci, means that the vision between the foci remains blurred. The EDOF IOL allows for a continuous range of vision and provides a consistent visual performance from far to intermediate distance. Due to the elongated focus this IOL is also less susceptible to errors in power calculation or the final position of the IOL, and the distance vision is quite well preserved despite minimal refractive error or remaining astigmatism [9]. In fact, our study showed that bilateral implantation of an EDOF lens provided excellent binocular uncorrected distance visual acuity despite the mean postoperative refractive error of $-0.41 \mathrm{D}$. The mean decimal UDVA was 1.04 (-0.02 logMAR) and $97.92 \%$ of the patients achieved 0.8 (0.1 logMAR) or better. This result was similar or superior to previous studies [6-11]. Pedrotti et al. reported a mean binocular UDVA of 0.08 (logMAR) [7]. A previous study which compared Tecnis Symfony with two trifocal IOLs reported a mean binocular UDVA of 0.833 (0.08 $\log$ MAR) which was comparable to the trifocal IOLs [10]. Another study on the Symfony IOL reported an UDVA of 0.03 ( $\log$ MAR) [11]. Mean UDVA of the non-monovision group in the multicenter Concerto study was 0.95 which was similar to 0.94 in the monovision group of the same study [6]. This shows that the residual refractive error does not significantly reduce uncorrected distance vision after EDOF IOL implantation. In our study, Symfony EDOF IOLs also showed excellent intermediate VA with a mean binocular UIVA of 0.96 (0.02 logMAR) and 93.75\% of patients achieved 0.8 (0.1 logMAR) or better. Patient satisfaction was highest for intermediate vision. Previous studies reported comparable intermediate VA of 0.79 and 0.99 at the same distance of $70 \mathrm{~cm}$ [6-12]. This shows that the Symfony IOL provides superior visual acuity across intermediate and far distances. Mean UNVA was 0.68 (0.17 logMAR) in our study which was lower than the intermediate and distance VA. This result is consistent with previous studies with Symfony IOLs targeting postoperative emmetropia [10-13]. However, $76.04 \%$ of the patients achieved an UNVA of $0.63(0.2$ $\operatorname{logMAR}$ ) or better which may be sufficient to provide functional vision for near tasks. Since this EDOF IOL provides an elongated focus and has no near addition, there is inevitably a theoretical loss of near vision. The extended range of focus may contribute to near visual function to some extent, as shown by a previous study which showed better near VA of Symfony IOLs compared to monofocal IOLs [7], but this may not be as efficient as a near addition. In fact, our study showed that more patients needed glasses for reading compared to far or intermediate distances. Previous studies have also acknowledged this limitation and suggested that this can be overcome with micromonovision [6-14].

More than $85 \%$ of our patients reported no or only mild halos, glare, and starbursts. This was similar to the Concerto study in which more than $90 \%$ reported no or mild photic phenomena [6]. Previous studies reported no significant difference in photic phenomena between Symfony EDOF and monofocal IOLs [7-13]. Although some of the other studies reported a much lower incidence of night-time visual disturbances, such as less than $1 \%$ [10], the difference is likely due to differences in questioning methods and lack of standardization in detecting visual symptoms. There were no cases of IOL explanation due to visual discomfort or dissatisfaction, and overall patient satisfaction was high, suggesting that visual symptoms likely had little or no impact on their daily activities. The limitations of this study include the difference in the methods of outcome measurement in terms of visual acuity and questionnaire on visual symptoms, which limits the direct comparison with the findings of previously published studies. Outcomes regarding photic phenomena and spectacle independence were self-reported and were at risk of recall bias. Also, only binocular VA was measured instead of both monocular and binocular VA. However, we think that binocular summation better reflects the circumstances in which a person usually functions.

\section{Conclusion}

The Symfony EDOF IOL demonstrated excellent distance and intermediate visual performance and functional near visual acuity. 
The visual results were associated with high levels of spectacle independence and patient satisfaction. Therefore, the Symfony IOL may be considered a promising option for cataract patients who want to achieve a consistently high quality of vision across far and intermediate distances.

\section{Acknowledgement}

None

\section{Conflict of interest}

The authors report no conflict of interest.

\section{Funding}

This research was supported by a grant of the Korea Health Technology R\&D Project through the Korea Health Industry Development Institute (KHIDI), funded by the Ministry of Health \& Welfare, Republic of Korea (grant number: HI18C1111). The Ministry of Health \& Welfare, Republic of Korea had no role in design and conduct of the study; collection, management, analysis, and interpretation of the data; and preparation, review, or approval of the manuscript.

\section{Author's contributions}

M.C., and T.K. conceived and designed the study. M.C., C.Y.I., J.K.L., H.K., H.S.P., and T.K. acquired the data. M.C., C.Y.I., J.K.L., H.K., H.S.P., and T.K. contributed in data analysis and interpretation. M.C., and T.K. prepared the manuscript. All authors reviewed and approved of the manuscript.

\section{References}

1. Lane SS, Morris M, Nordan L, Packer M, Tarantino N, et al. (2006) Multifocal intraocular lenses. Ophthalmol Clin North Am 19(1): 89-105.

2. Alba Bueno F, Vega F, Millan MS (2014) [Halos and multifocal intraocular lenses: origin and interpretation]. Arch Soc Esp Oftalmol 89(10): 397-404.
3. Alfonso JF, Fernandez Vega L, Amhaz H, Montes Mico R, Valcarcel B, et al. (2009) Visual function after implantation of an aspheric bifocal intraocular lens. J Cataract Refract Surg 35(5): 885-892.

4. Alfonso JF, Puchades C, Fernandez Vega L, Montes Mico R, Valcarcel B, et al. (2009) Visual acuity comparison of 2 models of bifocal aspheric intraocular lenses. J Cataract Refract Surg 35(4): 672-676.

5. Gatinel D, Pagnoulle C, Houbrechts Y, Gobin L (2011) Design and qualification of a diffractive trifocal optical profile for intraocular lenses. J Cataract Refract Surg 37(11): 2060-2067.

6. Cochener B, Concerto Study G (2016) Clinical outcomes of a new extended range of vision intraocular lens: International Multicenter Concerto Study. J Cataract Refract Surg 42(9): 1268-1275.

7. Pedrotti E, Bruni E, Bonacci E, Badalamenti R, Mastropasqua R, et al. (2016) Comparative Analysis of the Clinical Outcomes with a Monofocal and an Extended Range of Vision Intraocular Lens. J Refract Surg 32(7): 436-442.

8. Madrid Costa D, Ruiz Alcocer J, Ferrer Blasco T, Garcia Lazaro S, Montes Mico R (2013) Optical quality differences between three multifocal intraocular lenses: bifocal low add, bifocal moderate add, and trifocal. J Refract Surg 29(11): 749-754.

9. Akella SS, Juthani VV (2018) Extended depth of focus intraocular lenses for presbyopia. Curr Opin Ophthalmol 29(4): 318-322.

10. Cochener B, Boutillier G, Lamard M, Auberger Zagnoli C (2018) A Comparative Evaluation of a New Generation of Diffractive Trifocal and Extended Depth of Focus Intraocular Lenses. J Refract Surg 34(8): 507-514.

11. Attia MSA, Auffarth GU, Kretz FTA (2017) Clinical Evaluation of an Extended Depth of Focus Intraocular Lens with the Salzburg Reading Desk. J Refract Surg 33(10): 664-669.

12. Sachdev GS, Ramamurthy S, Sharma U, Dandapani R (2018) Visual outcomes of patients bilaterally implanted with the extended range of vision intraocular lens: A prospective study. Indian J Ophthalmol 66(3): 407-410.

13. Pedrotti E, Carones F, Aiello F (2018) Comparative analysis of visual outcomes with 4 intraocular lenses: Monofocal, multifocal, and extended range of vision. J Cataract Refract Surg 44(2): 156-167.

14. Ganesh S, Brar S, Pawar A, Relekar KJ (2018) Visual and Refractive Outcomes following Bilateral Implantation of Extended Range of Vision Intraocular Lens with Micromonovision. J Ophthalmol 2018: 7321794.

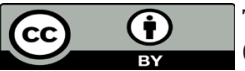

This work is licensed under Creative Commons Attribution 4.0 License

To Submit Your Article Click Here:

Submit Article

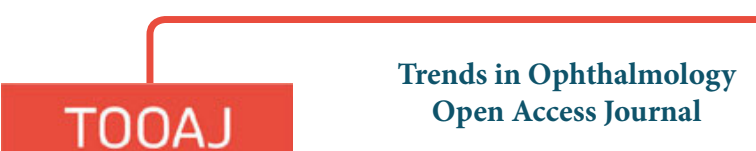

Assets of Publishing with us

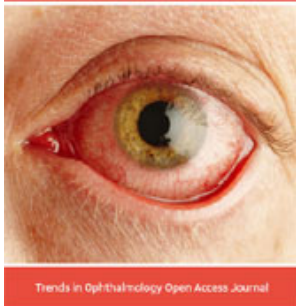

- Global archiving of articles

- Immediate, unrestricted online access

- Rigorous Peer Review Process

- Authors Retain Copyrights

- Unique DOI for all articles 
